\title{
Some Mean Inequalities
}

\author{
FINBARR HOLLAND
}

Dedicated to Trevor West on the occasion of his retirement.

Abstract. Let $\mathbb{P}$ denote the collection of positive sequences defined on the set of natural numbers $\mathbb{N}$. It is proved that if $x \in \mathbb{P}$, and $s<0$, then

$$
\frac{1}{n} \sum_{k=1}^{n}\left(\frac{1}{k} \sum_{j=1}^{k} x_{j}^{1 / s}\right)^{s} \leq\left(\frac{1}{n} \sum_{j=1}^{n}\left(\frac{1}{j} \sum_{k=1}^{j} x_{k}\right)^{1 / s}\right)^{s}, \quad n \in \mathbb{N}
$$

with equality if and only if $x$ is a constant sequence. This is a sharp refinement of an inequality discovered by Knopp in 1928.

\section{INTRODUCTION}

When I received the invitation to participate in the Westfest, I was in the throes of writing up a solution to the following problem, due to Joel Zinn, which was posed in the American Mathematical Monthly, and I offered to speak on this topic at the meeting in TCD to mark Trevor's retirement. I'm grateful to the organising committee of the Westfest for giving me the opportunity to do so.

Problem 1 (Number 11145). Find the least $c$ such that if $n \geq 1$, $a_{1}, \ldots, a_{n}>0$, then

$$
\sum_{k=1}^{n} \frac{k}{\sum_{j=1}^{k} \frac{1}{a_{j}}} \leq c \sum_{k=1}^{n} a_{k}
$$

I propose to describe a method to handle a family of similar problems of which this, and classical ones due to Carleman, and Knopp, are special cases. 


\section{BACKGROUND}

We denote by $\mathbb{P}$ the collection of positive sequences $x: \mathbb{N} \rightarrow(0, \infty)$. Clearly, $\mathbb{P}$ is a convex set. It is closed under the usual pointwise operations of addition and multiplication, and ordered by the relation:

$$
x \leq y \Longleftrightarrow x_{n} \leq y_{n}, \forall n \in \mathbb{N} .
$$

In particular, $\mathbb{P}$ is a commutative group under multiplication, with the sequence vector $e$ of ones acting as the identity. We'll write $1 / x$ for the multiplicative inverse of $x \in \mathbb{P}$ :

$$
(1 / x)_{n}=\frac{1}{x_{n}}, \forall n \in \mathbb{N}
$$

We recall a number of familiar functions that take $\mathbb{P}$ into itself:

$$
\begin{gathered}
A: \mathbb{P} \rightarrow \mathbb{P} ; \quad A(x)_{n}=\frac{1}{n} \sum_{k=1}^{n} x_{k}, n=1,2, \ldots ; \\
G: \mathbb{P} \rightarrow \mathbb{P} ; \quad G(x)_{n}=\sqrt[n]{\prod_{k=1}^{n} x_{k}}, n=1,2, \ldots ; \\
H: \mathbb{P} \rightarrow \mathbb{P} ; \quad H(x)_{n}=\frac{n}{\sum_{k=1}^{n} \frac{1}{x_{k}}}, n=1,2, \ldots ; \\
\min : \mathbb{P} \rightarrow \mathbb{P} ; \min (x)_{n}=\min \left\{x_{k}: k=1,2, \ldots, n\right\} .
\end{gathered}
$$

These are, respectively, the arithmetic, geometric, harmonic and minimum means. (Weighted versions of these exist, but I'll not have any need to refer to them.)

It is a well-known fact [5] that

$$
\min (x)_{n} \leq \frac{n}{\sum_{k=1}^{n} \frac{1}{x_{k}}} \leq \sqrt[n]{\prod_{k=1}^{n} x_{k}} \leq \frac{1}{n} \sum_{k=1}^{n} x_{k}, n=1,2, \ldots
$$

Moreover, the inequalities are strict unless $x$ is a constant sequence. Equivalently,

$$
\min \leq H \leq G \leq A .
$$

It's clear from the definitions that $A, G, H$ and min are "homogeneous" in the sense that, if $f \in\{A, G, H, \min \}$, then

$$
f(\lambda x)=\lambda f(x), \quad \forall x \in \mathbb{P}, \lambda>0 .
$$


It's perhaps less obvious, but nonetheless true, that they are superadditive: if $f \in\{A, G, H, \min \}$, then

$$
f(x)+f(y) \leq f(x+y), \forall x, y \in \mathbb{P} .
$$

Hence they are also concave on $\mathbb{P}$.

We also introduce a one-parameter family of functions $\left\{M_{t}: t>0\right\}$ that leave $\mathbb{P}$ invariant. If $x \in \mathbb{P}$, we define $M_{t}(x)$ by

$$
M_{t}(x)_{n}=\left(\frac{n}{\sum_{j=1}^{n} \frac{1}{x_{j}^{1 / t}}}\right)^{t}, n=1,2, \ldots,
$$

so that $M_{t}(x)=\left(H\left(x^{1 / t}\right)\right)^{t}$,

$$
\min (x) \leq M_{t}(x) \leq G(x) \leq A(x), \forall x \in \mathbb{P}, \forall t>0,
$$

and

$$
\lim _{t \rightarrow 0^{+}} M_{t}(x)=\min (x), \lim _{t \rightarrow \infty} M_{t}(x)=G(x), \forall x \in \mathbb{P} .
$$

\section{An Inequality Between Compositions of Means}

I'm interested in compositional relationships between these various functions. I'll describe the following result.

Theorem 1. Let $t>0$. Then $A \circ M_{t} \leq M_{t} \circ A$. Moreover, $A \circ$ $M_{t}(x)=M_{t} \circ A(x)$ if and only if $x=\lambda e$ for some $\lambda>0$.

For instance, when $t=1$, the claim is that $A \circ H \leq H \circ A$. Equivalently,

$$
\frac{1}{n} \sum_{k=1}^{n} \frac{k}{\sum_{j=1}^{k} \frac{1}{x_{j}}} \leq \frac{n}{\sum_{k=1}^{n} \frac{k}{\sum_{j=1}^{k} x_{j}}}, n=1,2, \ldots
$$

Even for small values of $n$ this is already fairly challenging, as the reader may discover for him or her self by considering the special case $n=3$.

A more general weighted inequality of this kind was first postulated by Nanjundiah [13] in 1952, but he offered no proof, and indeed his conjecture is not true generally. A special case of it was conjectured by myself [6] in 1992, and Kedlaya [8] supplied a proof of this in 1994, namely that $A \circ G \leq G \circ A$. In 1996, Mond and Pečarić [12] proved an analogue of the inequality $A \circ H \leq H \circ A$, the case $t=1$, for Hermitian matrices.

To establish the theorem, we begin by proving a lemma. 
Lemma 1. Let $t>0$ and let $p=t+1$. Let $x \in \mathbb{P}$. Then, for each $n \geq 1$,

$$
M_{t}(x)_{n}=n^{t} \inf \left\{\sum_{k=1}^{n} x_{k} a_{k}^{p}: 0<a \in \mathbb{R}^{n}, \sum_{k=1}^{n} a_{k}=1\right\} .
$$

Proof. Suppose $0<a \in \mathbb{R}^{n}$, and $\sum_{k=1}^{n} a_{k}=1$. Let $q=p /(p-1)=$ $p / t$. Then, by Hölder's inequality,

$$
\begin{aligned}
1 & =\sum_{j=1}^{n}\left(a_{j}^{p} x_{j}\right)^{1 / p} x_{j}^{-1 / p} \\
& \leq\left(\sum_{j=1}^{n} a_{j}^{p} x_{j}\right)^{1 / p}\left(\sum_{j=1}^{n} x_{j}^{-q / p}\right)^{1 / q},
\end{aligned}
$$

Hence

$$
\frac{1}{\left(\sum_{j=1}^{n} x_{j}^{-q / p}\right)^{p / q}} \leq \sum_{j=1}^{n} a_{j}^{p} x_{j} .
$$

Equality holds here if

$$
a_{j}=\frac{1}{x_{j}^{q / p}\left(\sum_{j=1}^{n} x_{j}^{-q / p}\right)}, j=1,2, \ldots, n .
$$

It follows that

$$
\begin{aligned}
\frac{1}{\left(\sum_{j=1}^{n} \frac{1}{x_{j}^{1 / t}}\right)^{t}}= & \frac{1}{\left(\sum_{j=1}^{n} x_{j}^{-q / p}\right)^{p / q}} \\
& =\inf \left\{\sum_{k=1}^{n} x_{k} a_{k}^{p}: 0<a \in \mathbb{R}^{n}, \sum_{k=1}^{n} a_{k}=1\right\} .
\end{aligned}
$$

The stated result follows.

An equivalent formulation is that, with $p=t+1$,

$$
M_{t}(x)_{n}=n^{p-1} \inf \left\{\sum_{k=1}^{n} x_{k} a_{k}^{p}: 0<a \in \mathbb{R}^{n}, \sum_{k=1}^{n} a_{k}=1\right\} .
$$

Thus

$$
M_{t}(x)_{n} \leq n^{p-1} \sum_{k=1}^{n} x_{k} a_{k}^{p}
$$

for all probability vectors $a \in \mathbb{R}^{n}$. 
Remark. Already this result reveals that $M_{t}$ is super-additive and hence concave.

The result we want to prove is the following: if $x \in \mathbb{P}$,

$$
\begin{aligned}
\frac{1}{n} \sum_{k=1}^{n}\left(\frac{k}{\sum_{j=1}^{k} \frac{1}{x_{j}^{1 / t}}}\right)^{t} & =\frac{1}{n} \sum_{k=1}^{n} M_{t}(x)_{k} \leq M_{t}(A(x))_{n} \\
& =\left(\frac{n}{\sum_{k=1}^{n} \frac{1}{A(x)_{k}^{1 / t}}}\right)^{t}, n=1,2, \ldots,
\end{aligned}
$$

with equality if and only if $x$ is a constant sequence.

Our idea is this: with $n$ fixed, suppose $a$ is a probability vector in $\mathbb{R}^{n}$. Then, by the previous lemma, with $p=t+1$,

$$
\begin{aligned}
M_{t}(A(x))_{n} & \leq n^{p-1} \sum_{j=1}^{n} a_{j}^{p} A(x)_{j} \\
& =n^{p-1} \sum_{j=1}^{n} \frac{a_{j}^{p}}{j} \sum_{k=1}^{j} x_{k} \\
& =n^{p-1} \sum_{k=1}^{n} x_{k} \sum_{j=k}^{n} \frac{a_{j}^{p}}{j},
\end{aligned}
$$

after interchanging the order of summation, and there is equality here for a suitable $a$. But, also, if $u_{i} \in \mathbb{R}^{i}$ is a probability vector,

$$
M_{t}(x)_{i} \leq i^{p-1} \sum_{j=1}^{i} u_{i j}^{p} x_{j}, i=1,2, \ldots, n,
$$

whence

$$
\begin{aligned}
\sum_{i=1}^{n} M_{t}(x)_{i} & \leq \sum_{i=1}^{n} i^{p-1} \sum_{j=1}^{i} u_{i j}^{p} x_{j} \\
& =\sum_{j=1}^{n} x_{j} \sum_{i=j}^{n} i^{p-1} u_{i j}^{p}
\end{aligned}
$$

So, we can accomplish our objective if, given a probability vector $a \in \mathbb{R}^{n}$, we can construct similar vectors $u_{i} \in \mathbb{R}^{i}$ so that

$$
\sum_{i=j}^{n} i^{p-1} u_{i j}^{p} \leq n^{p} \sum_{k=j}^{n} \frac{a_{k}^{p}}{k}, j=1,2, \ldots, n .
$$


To reach our goal, and to show that these inequalities can be solved, we construct a certain lower triangular row-stochastic matrix from a probability vector. To this end, we use the following result due to Kedlaya [8]:

Lemma 2. The rational numbers

$$
\alpha_{k}(i, j)=\frac{\left(\begin{array}{c}
n-i \\
j-k
\end{array}\right)\left(\begin{array}{c}
i-1 \\
k-1
\end{array}\right)}{\left(\begin{array}{c}
n-1 \\
j-1
\end{array}\right)}, 1 \leq i, j, k \leq n,
$$

satisfy the following conditions

(1) $\alpha_{k}(i, j) \geq 0$, for all $i, j, k$;

(2) $\alpha_{k}(i, j)=0$ for all $k>\min (i, j)$;

(3) $\alpha_{k}(i, j)=\alpha_{k}(j, i)$ for all $i, j, k$;

(4) $\sum_{k=1}^{n} \alpha_{k}(i, j)=1$ for all $i, j$;

(5) $\quad \sum_{i=1}^{n} \alpha_{k}(i, j)=\left\{\begin{array}{lc}\frac{n}{j}, & \text { for } 1 \leq k \leq j, \\ 0, & \text { fork }>j .\end{array}\right.$

Given a probability vector $a \in \mathbb{R}^{2}$, construct the $n \times n$ matrix $A=\left[a_{i j}\right]$ by

$$
a_{i j}=\sum_{k=1}^{n} \alpha_{j}(i, k) a_{k}, 1 \leq i, j \leq n .
$$

Then each row of $A$ is a probability vector, because

$$
\begin{aligned}
\sum_{j=1}^{n} a_{i j} & =\sum_{j=1}^{n} \sum_{k=1}^{n} \alpha_{j}(i, k) a_{k} \\
& =\sum_{k=1}^{n} a_{k} \sum_{j=1}^{n} \alpha_{j}(i, k) \\
& =\sum_{k=1}^{n} a_{k} \text { (by 4.) } \\
& =1
\end{aligned}
$$

for all $i$. Also, $a_{i j}=0$ for all $j>i$. Thus $A$ is a lower triangular row-stochastic matrix.

But, for each pair of indices $i, j, a_{i j}$ is a convex combination of $a_{1}, a_{2}, \ldots, a_{n}$, and so, if $p \geq 1$,

$$
a_{i j}^{p} \leq \sum_{k=1}^{n} \alpha_{j}(i, k) a_{k}^{p}, i, j=1,2, \ldots, n .
$$


Hence

$$
\begin{aligned}
\sum_{i=j}^{n} i^{p-1} a_{i j}^{p} & =\sum_{i=1}^{n} i^{p-1} a_{i j}^{p} \\
& \leq n^{p-1} \sum_{i=1}^{n} \sum_{k=1}^{n} \alpha_{j}(i, k) a_{k}^{p} \\
& =n^{p-1} \sum_{k=1}^{n} a_{k}^{p} \sum_{i=1}^{n} \alpha_{j}(i, k) \\
& =n^{p} \sum_{k=j}^{n} \frac{a_{k}^{p}}{k} \text { (by 5.). }
\end{aligned}
$$

Looking back at (3) we now see that we can solve this by selecting

$$
u_{i j}=a_{i j}, j=1,2, \ldots, i .
$$

We're now ready to provide a proof of the theorem.

Fix $x \in \mathbb{P}$, and a positive integer $n$. Let $a$ be a probability vector in $\mathbb{R}^{n}$. Choose the corresponding lower triangular row-stochastic matrix $A=\left[a_{i j}\right]$ as above. By Lemma 1 , if $1 \leq i \leq n$,

$$
M_{t}(x)_{i} \leq i^{p-1} \sum_{j=1}^{i} a_{i j}^{p} x_{j} .
$$

Hence

$$
\begin{aligned}
\sum_{i=1}^{n} M_{t}(x)_{i} & \leq \sum_{i=1}^{n} i^{p-1} \sum_{j=1}^{i} a_{i j}^{p} x_{j} \\
& =\sum_{j=1}^{n} x_{j} \sum_{i=j}^{n} i^{p-1} a_{i j}^{p} \\
& \leq \sum_{j=1}^{n} x_{j} n^{p} \sum_{k=j}^{n} \frac{a_{k}^{p}}{k} \\
& =n^{p} \sum_{k=1}^{n} a_{k}^{p} \frac{1}{k} \sum_{j=1}^{k} x_{j} \\
& =n^{p} \sum_{k=1}^{n} a_{k}^{p} A(x)_{k} .
\end{aligned}
$$


Whence

is a lower bound for the set

$$
n^{-t-1} \sum_{i=1}^{n} M_{t}(x)_{i}
$$

$$
\left\{\sum_{k=1}^{n} a_{k}^{p} A(x)_{k}: 0<a \in \mathbb{R}^{n}, \sum_{k=1}^{n} a_{k}=1\right\},
$$

whose infimum is $n^{-t} M_{t}(A(x))_{n}$. Hence

$$
\frac{1}{n} \sum_{i=1}^{n} M_{t}(x)_{i} \leq M_{t}(A(x))_{n},
$$

and we're done, apart from dealing with the case of equality, which is easily settled.

\section{A Number of Corollaries}

We deduce a number of special cases of Theorem 1 .

\section{Corollary 1.}

$$
A \circ \min \leq \min \circ A .
$$

This is obtained by letting $t \rightarrow 0^{+}$.

Corollary 2 (Kedlaya).

$$
A \circ G \leq G \circ A \text {. }
$$

i.e., $\forall x \in \mathbb{P}$,

$$
\frac{1}{n} \sum_{i=1}^{n} G(x)_{i} \leq G(A(x))_{n}, \forall x \in \mathbb{P}, \forall n \geq 1 ;
$$

or, more explicitly,

$$
\frac{1}{n} \sum_{i=1}^{n} \sqrt[i]{\prod_{j=1}^{i} x_{j}} \leq \sqrt[n]{\prod_{j=1}^{n} \frac{1}{j} \sum_{i=1}^{j} x_{i}}
$$

This is obtained by letting $t \rightarrow \infty$. This implies Carleman's classical inequality $[3,5,7]$ :

$$
\|G(x)\|_{1} \leq e\|x\|_{1}, \forall x \in \mathbb{P} .
$$

Corollary 3 (Mond \& Pečarić).

$$
A \circ H \leq H \circ A \text {. }
$$


This is obtained by letting $t=1$. It says that, $\forall x \in \mathbb{P}$,

$$
\frac{1}{n} \sum_{k=1}^{n} \frac{k}{\sum_{j=1}^{k} \frac{1}{x_{j}}} \leq \frac{n}{\sum_{k=1}^{n} \frac{k}{\sum_{j=1}^{k} x_{j}}}, n=1,2, \ldots
$$

Since the sequence

$$
\sum_{j=1}^{k} x_{j}, k=1,2, \ldots
$$

is strictly increasing we deduce that the right-hand side does not exceed

whence

$$
\frac{n \sum_{j=1}^{n} x_{j}}{\sum_{k=1}^{n} k}=\frac{2 \sum_{j=1}^{n} x_{j}}{n+1}
$$

$$
\sum_{k=1}^{n} \frac{k}{\sum_{j=1}^{k} \frac{1}{x_{j}}} \leq \frac{2 n}{n+1} \sum_{j=1}^{n} x_{j}<2 \sum_{j=1}^{n} x_{j},
$$

which gives a solution to Zinn's Monthly problem. Moreover, the constant 2 cannot be replaced by a smaller number, as can be seen by taking $x_{j}=1 / j, j=1,2, \ldots, n$. Thus, if $x \in \mathbb{P} \cap \ell_{1}$, so does $H(x)$, and $\|H(x)\|_{1}<2\|x\|_{1}$.

Corollary 4. $\forall t>0$ and $\forall x \in \mathbb{P}$,

$$
\sum_{i=1}^{n} M_{t}(x)_{i} \leq\left(\frac{n^{1+1 / t}}{\sum_{j=1}^{n} j^{1 / t}}\right)^{t} \sum_{k=1}^{n} x_{k}, n=1,2, \ldots
$$

and the inequality is strict unless $n=1$.

Proof.

$$
\begin{aligned}
n M_{t}(A)_{n} & =n^{p}\left(\frac{1}{\sum_{j=1}^{n} A_{j}^{-1 / t}}\right)^{t} \\
& =n^{p}\left(\frac{1}{\sum_{j=1}^{n} \frac{j^{1 / t}}{\left(\sum_{k=1}^{j} x_{k}\right)^{1 / t}}}\right)^{t} \\
& \leq n^{p} \sum_{k=1}^{n} x_{k}\left(\frac{1}{\sum_{j=1}^{n} j^{1 / t}}\right)^{t} \\
& =\left(\frac{n^{1+1 / t}}{\sum_{j=1}^{n} j^{1 / t}}\right)^{t} \sum_{k=1}^{n} x_{k} .
\end{aligned}
$$


Since

$$
\lim _{n \rightarrow \infty} \frac{n^{1+1 / t}}{\sum_{j=1}^{n} j^{1 / t}}=1+\frac{1}{t}
$$

a simple consequence of the fact that, with $s=1 / t$,

$$
\frac{\sum_{j=1}^{n} j^{s}}{n^{s+1}}=\frac{1}{n} \sum_{j=1}^{n}\left(\frac{j}{n}\right)^{s}
$$

is a Riemann sum for the integral

$$
\int_{0}^{1} x^{s} d x=\frac{1}{1+s}
$$

Corollary 4 implies a result of Knopp [10] to the effect that

$$
\left\|M_{t}(x)\right\|_{1} \leq(1+1 / t)^{t}\|x\|_{1}, \forall x \in \mathbb{P} .
$$

\section{Companion Results When $t<0$}

The means $M_{t}$ also make sense when $t<0$. Similar methods to those employed in the previous section lead to the following statement.

Theorem 2. If $-1<t<0$, then $A \circ M_{t} \geq M_{t} \circ A$. Moreover, $A \circ M_{t}(x)=M_{t} \circ A(x)$ if and only if $x=\lambda e$ for some $\lambda>0$.

Letting $p=-1 / t$, we can recast this in terms of $p$ : If $p \geq 1$, then, for all $x \in \mathbb{P}$, and all $n \geq 1$,

$$
\left(\frac{1}{n} \sum_{k=1}^{n}\left(\frac{1}{k} \sum_{i=1}^{k} x_{i}\right)^{p}\right)^{1 / p} \leq \frac{1}{n} \sum_{k=1}^{n}\left(\frac{1}{k} \sum_{i=1}^{k} x_{i}^{p}\right)^{1 / p}
$$

There is equality only when $x$ is a constant sequence. This is a substantial improvement of a very well-known result due to Hardy $[4,5]$, which states that, if $x \in \ell_{p}$, then $A(x) \in \ell_{p}$, and

$$
\|A(x)\|_{p} \leq \frac{p}{p-1}\|x\|_{p}
$$

Inequality (5) was found by Bennett [2], who pointed out that the reversed inequality holds when $0<p<1$. A stronger form of (5) was established by B. Mond and J. E. Pečarić [11], and a weighted version of their result was outlined by Kedlaya [9]. But results of this kind were announced much earlier by Nanjundiah [13], though he appears not to have published a proof. 


\section{REFERENCES}

[1] G. Bennetr, Factorizing the Classical Inequalities, Mem. Amer. Math. Soc. 120 (1996), no. 576, viii+130pp.

[2] G. Bennetr, Summability matrices and random walk, Houston J. Math 28 (2002), no. 4, 865-898.

[3] T. Carleman, Sur les functions quasi-analytiques, Fifth Scand. Math. Congress (1923), 181-196.

[4] G. H. HARDY, Notes on some points of the integral calculus (LX), Messenger of Math. 54 (1925), 150-156.

[5] G. H. Hardy, J. E. Littlewood, and G. Pólya, Inequalities, Cambridge University Press, 1934.

[6] F. Holland, On a mixed arithmetic-mean, geometric-mean inequality, Math. Competitions 5 (1992), 60-64.

[7] M. Johansson, L. E. Persson, A. Wedestig, Carleman's inequalityhistory, proofs and some new generalizations, J. Ineq. Pure ES Appl. Math. 4 (3), (2003), 1-19.

[8] K. Kedlaya, Proof of a mixed arithmetic-mean, geometric-mean inequality, Amer. Math. Monthly 101 (1994), 355-357.

[9] K. Kedlaya, A weighted mixed-mean inequality, Amer. Math. Monthly 106 (1999), 355-358.

[10] K. Knopp, Über Reihen mit positiven Gliedern, J. London Math. Soc., 3 (1928), 205-211.

[11] B. Mond And J. E. Pě̌arić, A mixed means inequality, Austral.Math. Soc. Gaz., 23(2) (1996), 67-70.

[12] B. MONd AND J. E. PEČARIĆ, A mixed arithmetic-mean-harmonic-mean inequality, Lin. Algebra Appl. 237/238 (1996), 449-454.

[13] T. S. Nanjundiah, Sharpening of some classical inequalities, Math. Student 20 (1952), 24-25.

Finbarr Holland,

Mathematics Department,

University College,

Cork, Ireland

f.holland@ucc.ie

Received on 8 June 2006. 